\title{
Prognóstico do Duke-Escore Versus Cintilografia em Pacientes com Fatores de Risco para Doença Arterial Coronariana. Seguimento de Um Ano
}

\author{
Prognosis of Duke Treadmill Score Versus Scintilography in Patients at Risk for Coronary \\ Artery Disease. One Year Follow-up
}

Carlos Alberto Gasperin, Caroline Pereira Bueno, Airton Seiji Yamada, Gilberto Alves da Rocha, Regina Célia Portugal Freixo Heinze, Débora Cristina Fontoura de Mello Nunes, Isabelle Vianna Trevisan, Deisi Raquel Mostiack, Lânia Romanzin Xavier, Cláudia Maria Castelo Branco Silva Centro de Radioimunoensaio e Medicina Nuclear do Paraná - CERMEN - Curitiba, PR

\section{ObJetivo}

Determinar a sensibilidade, a especificidade e o risco de eventos cardiovasculares em pacientes com Duke-escore (DE) baixo, intermediário e alto risco, em comparação com a presença (ou não) de defeitos de perfusão à cintilografia do miocárdio.

\section{Métodos}

Estudo prospectivo, consecutivo, com 173 pacientes com 2 ou mais fatores de risco para doença arterial coronariana (DAC), que foram submetidos à cintilografia de perfusão do miocárdio com tetrofosmin marcado com tecnécio-Tc 99m (CPM) e ao teste ergométrico (aplicando-se o DE), de um bairro de Curitiba, entre janeiro de 2003 a fevereiro de 2004. Os pacientes tiveram seguimento de $13 \pm 1$ meses e 162 completaram o acompanhamento. Foi avaliada a presença de morte, angina, infarto agudo do miocárdio, angioplastia coronariana e revascularização do miocárdio.

\section{Resultados}

A média do $D E$ dos pacientes que apresentaram eventos (18) foi de $-0,27(95 \% \mathrm{IC}=-3,97 \mathrm{a}+3,91)$ e daqueles livres de eventos (144) foi de $+4,92$ ( $95 \%$ $\mathrm{IC}=+4,03 \mathrm{a}+5,81)$, com $\mathrm{p}<0,00069$. A sensibilidade do DE foi de $72,22 \%$ e da CPM foi de $77,78 \%$, sem diferença estatística, com $p=0,21$. A especificidade do DE foi de $54,17 \%$ e a da CPM foi de $88,19 \%$, com $p<0,0001$. A curva de Kaplan-Meier demonstrou que $94 \%$ dos pacientes com DE baixo risco permaneceram livres de eventos em 01 ano. Em contraste, todos os de alto risco apresentaram eventos no mesmo período. Os que apresentaram DE de intermediário risco apresentaram $15 \%$ de eventos em 01 ano.

\section{CONCLUSÃo}

O DE foi tão sensível quanto CPM em determinar o risco para DAC em um ano. Os pacientes com $D E<-0,27$ tiveram maior risco de eventos cardíacos.

\section{Palavras-chave}

Exercício, teste, prognóstico, doença arterial coronariana.

\section{Objective}

The purpose was to determine the sensitivity, the specificity and a year-long risk of subsequent cardiovascular events in patients with low, intermediate and high risk prognostic Duke treadmill score (DTS) in comparison with the presence (or not) of the myocardial perfusion defects on radionuclide images.

\section{Methods}

A prospective study, with 173 consecutive patients with 02 or more risk factors to coronary artery disease $(C A D)$, who underwent to exercise single photon - emission computed tomographic myocardial perfusion images using technetium-99m tetrophosmin (SPECT) and treadmill test (with DTS), from one neighbourhood of Curitiba city, between January 2003 and February 2004, were followed up for cardiac-cause mortality and

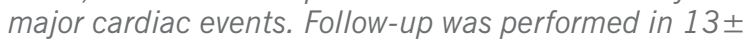
1 months and in 162 patients was complete.

\section{RESULTS}

The DTS mean those patients with cardiac event (18) over a year was $-0.27(95 \% \mathrm{Cl}=-3.97$ to +3.91$)$ and those free cardiac event patients (144) was +4.92 $(95 \% \mathrm{Cl}=+4.03$ to +5.81$)$, with $p<0.00069$. The DTS sensitivity was $72.22 \%$ and the SPECT sensitivity was $77.78 \%$, with no significant difference $p=0.21$. The DTS specificity was $54.17 \%$ and the SPECT specificity was $88.19 \%$, with $p<0.0001$. The cumulative proportion free-events (Kaplan-Meier) curves demonstrated that 94\% those patients with low-risk DTS remained freecardiac events. In contrast, all high-risk DTS had adverse cardiac events. Those patients with intermediate-risk DTS had $15 \%$ of cardiac event over a year.

\section{Conclusion}

The DTS was as sensitivity as SPECT in determine a year risk for CAD. Those patients with DTS <-0.27 had high-risk cardiac event.

\section{KEY WORDS}

Exercise, tests, prognosis, coronary artery disease

Correspondência: Carlos Alberto Gasperin - Rua Gonçalves Dias, 655 - 80240-340 - Curitiba, PR E-mail: acgasperin@cendicor.com.br Recebido em 11/10/05 - Aceito em 24/03/06 
Apesar dos avanços tecnológicos do teste ergométrico (TE), como filtros computadorizados que permitem um traçado com pouca interferência e dos inúmeros conhecimentos metodológicos adquiridos nos seus mais de sessenta anos de existência, este exame apresenta menor sensibilidade diagnóstica na detecção da doença arterial coronariana (DAC) do que a de outros métodos de estresse, como a cintilografia de perfusão do miocárdio, o ecocardiograma de estresse e a tomografia computadorizada do coração ${ }^{1}$. Recentemente, foram desenvolvidos índices prognósticos, como os índices de "Duke - escore"2 e o "Veterans - escore" ${ }^{3}$ aplicados ao TE com o objetivo de aumentar sua sensibilidade diagnóstica e prognóstica.

Nosso objetivo neste estudo foi o de comparar a sensibilidade e a especificidade do índice prognóstico do Duke-escore (DE) com a cintilografia de perfusão do miocárdio (Tc ${ }^{99 m}$-tetrofosmin) de estresse físico (CPM) num mesmo grupo de pacientes com fatores de risco para DAC, durante 12 meses.

\section{Métodos}

Foram alocados para o estudo 311 pacientes de forma consecutiva, encaminhados para exame de cintilografia do miocárdio, de um determinado bairro (Seminário) de Curitiba, Paraná, com dois ou mais fatores de risco para DAC, de janeiro de 2003 até fevereiro de 2004. Os fatores de risco considerados foram: hipertensão arterial sistêmica, diabete melito, dislipidemia, obesidade, doença arterial coronariana prévia, tabagismo e história familiar para coronariopatia. Todos os indivíduos foram informados quanto ao estudo e assinaram termo de consentimento. Foram considerados dentro do grupo com evento coronariano, os indivíduos que apresentaram, ao longo de 12 meses, internação hospitalar por angina pectoris, infarto agudo do miocárdio (IAM), angioplastia coronariana e revascularização do miocárdio, além de morte por IAM. Os demais foram considerados indivíduos sem evento coronariano.

Dos 311 indivíduos, 138 foram encaminhados para exame farmacológico (por: dificuldade de deambulação, bloqueio de ramo esquerdo, taquiarritmias ventriculares graves, fibrilação atrial ou portadores de marcapasso) e, em seguida, excluídos do estudo. Os demais 173 pacientes que realizaram teste ergométrico (protocolo de Bruce) e cintilografia de perfusão do miocárdio com tetrosfosmin marcado com tecnécio (Tc ${ }^{99 m}$ ), ambos no mesmo dia, foram incluídos no estudo. Desses, não foi possível obter o seguimento em 11 pacientes, os quais foram excluídos do estudo.

Os 162 pacientes restantes foram acompanhados de forma prospectiva, durante no mínimo 12 meses, para o objetivo de avaliar a presença dos seguintes eventos coronarianos: angina pectoris, infarto agudo do miocárdio, necessidade de angioplastia transluminal coronariana percutânea ou revascularização do miocárdio e morte.

O protocolo de esteira de Bruce foi aplicado a todos os indivíduos que realizaram a ergometria. 0 índice de Duke escore $^{2}$ do teste ergométrico foi obtido com a fórmula: tempo (minutos) - $5 \mathrm{X}$ (infradesnível de segmento ST mm) - $4 X$ (dor torácica: $0=$ sem dor/ $1=$ dor não limitante/ $2=$ dor limitante). Foi considerado Duke escore baixo risco o índice $\geq+5$, risco intermediário entre +4 a -10 e alto risco $\leq-11$. A análise de segmento ST foi obtida, sem o conhecimento dos dados clínicos do paciente, por 2 examinadores com experiência em ergometria e os resultados confrontados entre si, com a finalidade de se diminuírem erros subjetivos de análise. 0 tempo foi obtido pelo sistema computadorizado utilizado para realizar o teste ergométrico (Ergo-S/Dixtal). A análise de dor foi obtida por meio de questionário respondido pelo paciente durante o teste ergométrico, como descrito.

A cintilografia de perfusão do miocárdio com tetrofosmin marcado com tecnécio ( $T c^{99 m}$ ) foi obtida com protocolo de um dia. Inicialmente foi feita a imagem de repouso e, cerca de 6 horas depois, foi repetida a imagem após estresse físico em esteira ergométrica. Foram obtidas imagens tomográficas de 60 quadros com aquisição contínua no repouso. As imagens tomográficas após estresse físico foram sincronizadas ao eletrocardiograma do paciente, sendo o intervalo RR dividido em 8 quadros, totalizando 480 quadros por exame. Foi considerada como anormal a CPM que apresentou qualquer defeito de perfusão transitória de parede de ventrículo esquerdo (isquemia), qualquer defeito de perfusão persistente de parede de ventrículo esquerdo em paciente sem história prévia de IAM ou com qualquer hipocinesia associada ou não à fração de ejeção < 50\% à análise funcional, sem história prévia de IAM.

Foram determinadas as médias com desvio-padrão e erro-padrão entre os grupos com e sem eventos coronarianos para ambos os métodos (DE e CPM), utilizando-se o método " $t$ - student" para duas variáveis independentes. Para análise entre as variáveis de risco coronariano, foi utilizado o "teste de Fischer". Foram, ainda, determinadas a sensibilidade e especificidade prognósticas do DE e da CPM, comparadas entre si, aplicando-se o "teste entre duas proporções independentes". Por fim, foi determinada a curva livre de eventos coronarianos, pelo "método de sobrevivência de Kaplan-Meier". Foi considerado como estatisticamente significativo o índice de $p<0,05$.

\section{Resultados}

Os pacientes que apresentaram evento coronariano ao longo de 12 meses foram 18 e os que permaneceram livres de eventos totalizaram 144.

A tabela 1 mostra que o sexo masculino, a hipertensão arterial, o diabete melito e média maior de idade foram 
estatisticamente mais freqüentes no grupo com evento coronariano. Na tabela 2 demonstrou-se que não houve diferença estatisticamente significativa entre as proporções dos fatores de risco pesquisados entre as populações com DE baixo risco e intermediário/alto risco. No caso da cintilografia, na tabela 3 registrou-se que ocorreu maior porcentagem de casos do sexo masculino, maior número de hipertensos, diabéticos e com coronariopatia prévia no grupo com cintilografia alterada.

O número de pacientes com DE baixo, intermediário e alto, bem como o número de pacientes com CPM normal e alterada estão representados nas figuras 1 e 2 , respectivamente.
A média do DE obtida em pacientes livres de eventos coronarianos foi de +4,92 (IC95\%: +4,03 a $+5,81)$. A média do DE obtida no grupo de pacientes que apresentaram eventos coronarianos foi de $-0,27$ (IC95\%: $-3,97 a+3,91$ ). Como se demonstra na figura 3 , ocorreu diferença estatisticamente significativa entre a média do DE entre os dois grupos, com $p<0,00069$. Nesta figura, também se demonstram o erro-padrão e 0 desvio-padrão.

A sensibilidade do DE foi de $72,22 \%$ e da CPM foi de $77,78 \%$, porém, apesar da sensibilidade maior da CPM, não ocorreu significação estatística em relação ao $\mathrm{DE}, \operatorname{com} \mathrm{p}=0,21$.

\section{Tabela 1 - Comparação dos fatores de risco entre as populações com e sem eventos em 12 meses}

\begin{tabular}{lccc} 
& Sem evento $(\mathbf{n}=\mathbf{1 4 4})$ & Com evento $(\mathbf{n}=\mathbf{1 8})$ & $\mathbf{p}$ \\
Sexo masculino & $54,17 \%$ & $88,89 \%$ & $\mathrm{p}<0,04$ \\
Idade média (anos) & 56,05 & 62,61 & $\mathrm{p}=0,02$ \\
Hipertensão arterial & $61,11 \%$ & $88,89 \%$ & $\mathrm{p}=0,02$ \\
Diabete & $11,11 \%$ & $38,89 \%$ & $\mathrm{p}=0,005$ \\
Dislipidemia & $57,64 \%$ & $61,11 \%$ & $\mathrm{p}=1,0$ \\
Obesidade & $15,28 \%$ & $27,78 \%$ & $\mathrm{p}=0,18$ \\
História familiar para coronariopatia & $50 \%$ & $50 \%$ & $\mathrm{p}=1,0$ \\
\hline Tabagismo & $14,58 \%$ & $16,67 \%$ & $\mathrm{p}=0,72$ \\
IAM prévio & $6,84 \%$ & $11,11 \%$ & $\mathrm{p}=0,62$ \\
Angioplastia prévia & $18,75 \%$ & $27,78 \%$ & $\mathrm{p}=0,35$ \\
Revascularização prévia & $4,9 \%$ & $16,67 \%$ & $\mathrm{p}=0,08$
\end{tabular}

Tabela 2 - Comparação dos fatores de risco entre as populações com Duke -escore com baixo risco e com risco intermediário/alto

\begin{tabular}{lccc} 
& Duke baixo $(\mathbf{n = 8 3})$ & Duke interm/alto $(\mathbf{n = 7 9 )}$ & $\mathbf{p}$ \\
Sexo masculino & $63,88 \%$ & $51,90 \%$ & 0,15 \\
Idade média (anos) & 55,31 & 58,32 & 0,10 \\
Hipertensão arterial & $63,86 \%$ & $64,56 \%$ & 1,0 \\
Diabete & $9,64 \%$ & $18,99 \%$ & 0,11 \\
Dislipidemia & $57,83 \%$ & $58,23 \%$ & 1,0 \\
Obesidade & $20,48 \%$ & $12,66 \%$ & 0,20 \\
História familiar para coronariopatia & $45,78 \%$ & $54,43 \%$ & 0,34 \\
Tabagismo & $15,66 \%$ & $13,92 \%$ & 0,82 \\
IAM prévio & $8,43 \%$ & $6,33 \%$ & 0,76 \\
Angioplastia prévia & $18,07 \%$ & $21,52 \%$ & 0,69 \\
Revascularização prévia & $3,61 \%$ & $8,86 \%$ & 0,20 \\
\multicolumn{1}{|c|}{ IAM - infarto agudo do miocárdio. } & & &
\end{tabular}

Tabela 3 - Comparação dos fatores de risco entre as populações com cintilografia normal e alterada

Cintilo normal $(\mathrm{n}=131)$
$53,44 \%$
56,12
$59,00 \%$
$10,69 \%$
$58,78 \%$
$16,03 \%$
$48,09 \%$
$13,74 \%$
$1,53 \%$
$14,50 \%$
$2,29 \%$

Cintilo alterada $(\mathbf{n}=\mathbf{3 1})$
$77,42 \%$
59,54
$40,46 \%$
$29,03 \%$
$54,84 \%$
$19,35 \%$
$58,06 \%$
$19,35 \%$
$32,26 \%$
$41,94 \%$
$22,58 \%$

$\mathrm{p}$

0,01

0,15

0,01

0,018

0,69

0,60

0,42

0,41

$<0,001$

$<0,001$

$<0,001$ 


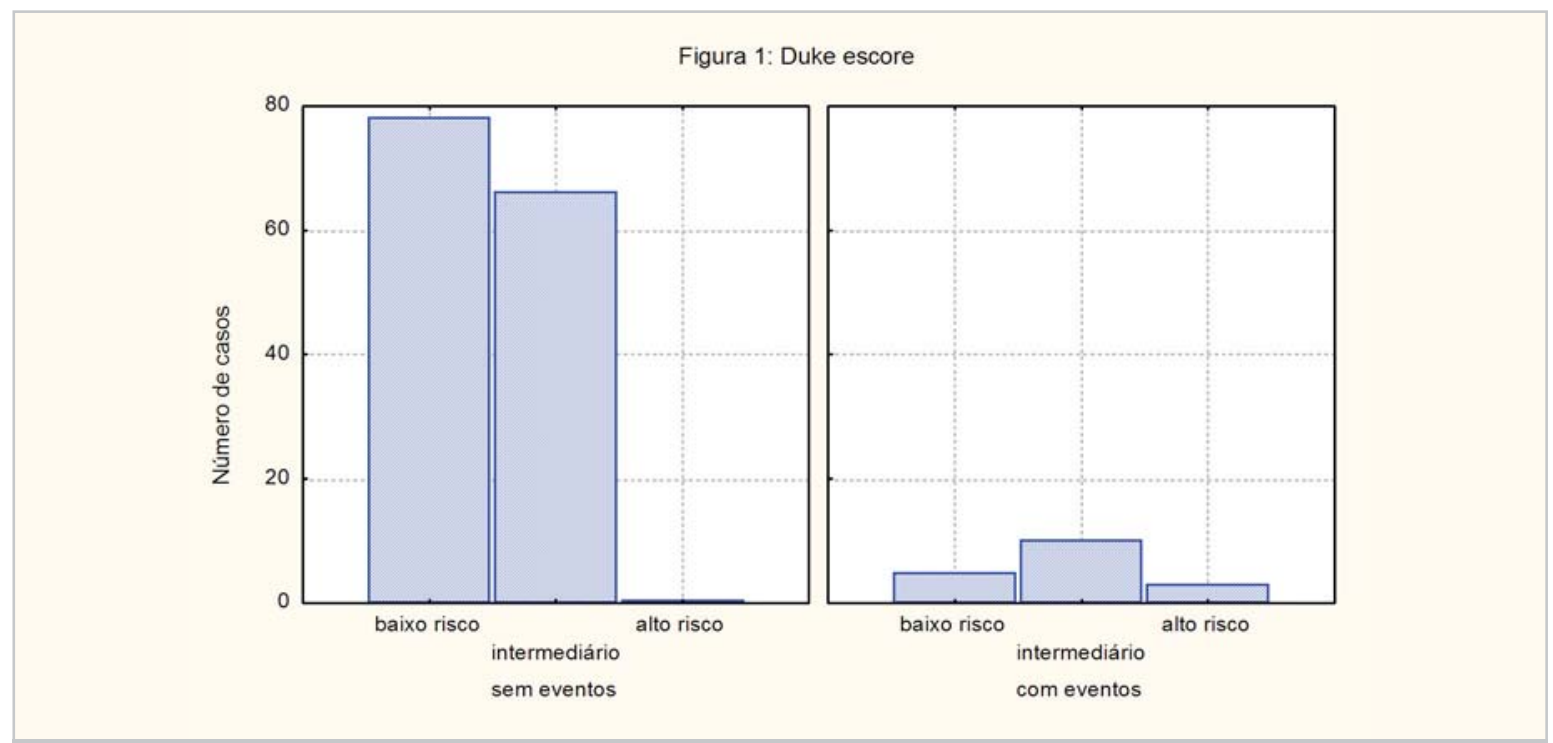

Fig. 1 - Duke-escore baixo, intermediário e alto - número de casos na população livre de eventos e que apresentaram eventos coronarianos.

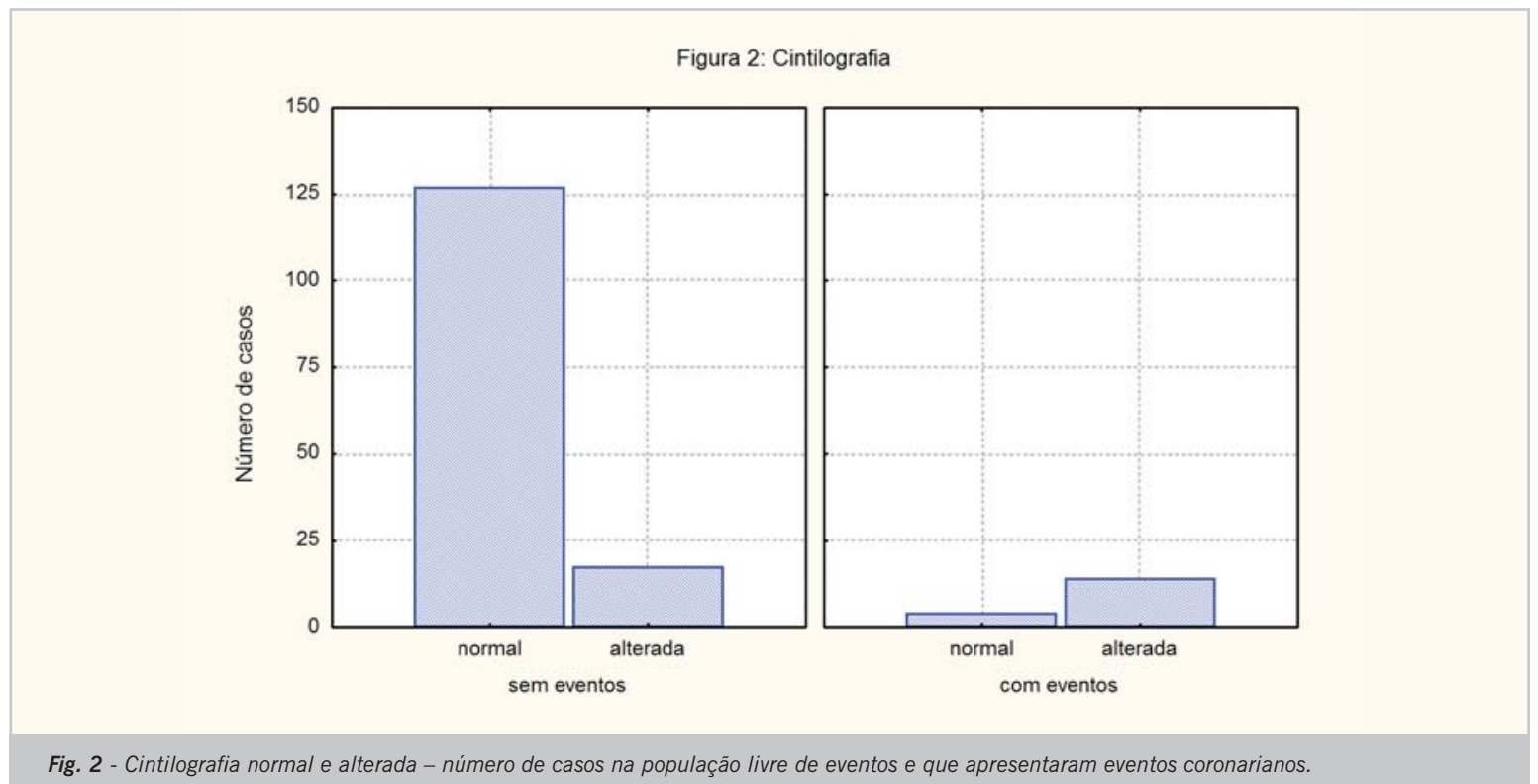

A especificidade do DE foi de $54,17 \%$ e da CPM foi de $88,19 \%$. A alta especificidade da CPM em relação ao DE apresentou relevância estatística, com $\mathrm{p}<00001$.

A média da fração de ejeção obtida pela análise funcional da CPM foi de 60,65\% nos pacientes livres de eventos em 12 meses e de 55,58\% nos que apresentaram evento no mesmo período; não houve relevância estatística entre os dois grupos, com $p=0,08$.

$\mathrm{Na}$ análise proporcional acumulada livre de eventos ao longo de 12 meses (curvas de Kaplan-Meier), observou-se nítida diferença entre os pacientes com CPM normal e CPM alterada $(p<0,0001)$, conforme se demonstra na figura 4 . Acima de $95 \%$ dos pacientes com CPM normal ficaram livres de eventos neste período, contra apenas 55\% dos pacientes com CPM anormal. A mesma análise para o DE, demonstrou que ao longo de 12 meses, cerca de $94 \%$ dos pacientes considerados de baixo risco ficaram livres de eventos, ao passo que todos os considerados alto risco tiveram eventos coronarianos $(p<0,0001)$. Cerca de $15 \%$ dos pacientes de risco intermediário apresentaram eventos no decorrer de 12 meses.

\section{Discussão}

Balady e cols. ${ }^{4}$ demonstraram em mais de 3.000 pacientes assintomáticos com fatores de risco para doença arterial coronariana, acompanhados num período superior a 18 anos, que o teste de esforço pôde auxiliar a detectar pessoas com maior risco de eventos, tanto em pacientes que apresentavam escore de Framingham de alto risco, quanto os de risco baixo. Três variáveis obtidas no teste ergométrico foram importantes: a depressão 

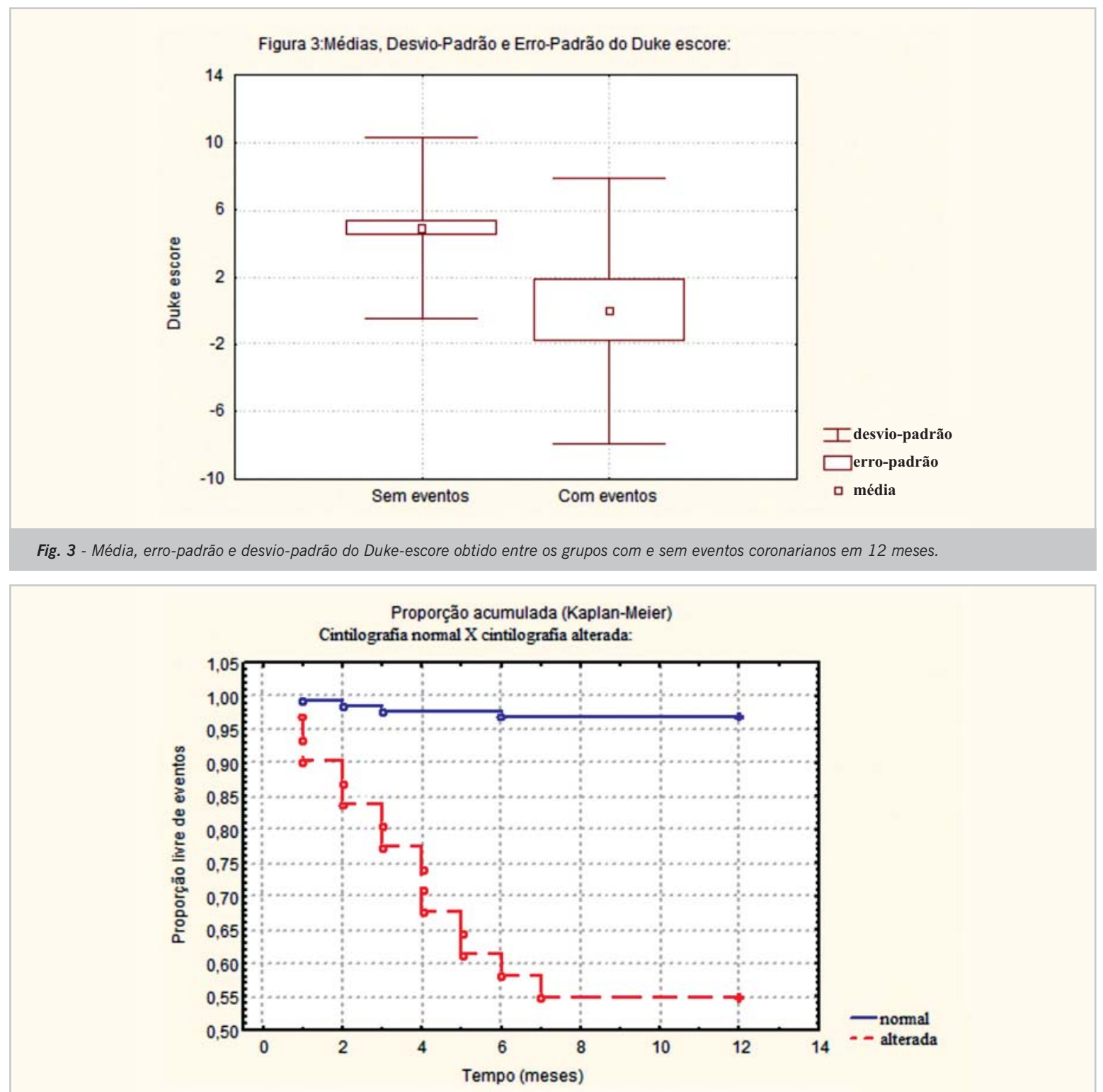

Fig. 4 - Comparação das curvas de Kaplan-Meier de proporção acumulada livre de eventos coronarianos entre o grupo de pacientes com cintilografia normal e alterada.

de segmento ST > $1 \mathrm{~mm}$, a capacidade de exercício em METs e a incapacidade de atingir a freqüência cardíaca submáxima prevista. Para cada MET acrescido ao desempenho no esforço, ocorreu uma redução de 13\% do risco de eventos. Vale notar que o infradesnivelamento de segmento ST e o número de METs atingido no esforço, dois dos dados mais significativos que, isoladamante, acrescentaram informações sobre o risco ao escore de Framingham, são também aplicados para obter o DE. O estudo acima demonstrou, de forma indireta e em grande população, o que o DE procura avaliar com o teste ergométrico de forma simples e expressa de forma numérica: o risco de evento coronariano do paciente. Até o momento, em nenhum estudo se comparou o prognóstico do DE com a CPM na população brasileira, o que motivou a realização deste estudo.
O dado mais importante dele foi a sensibilidade do $\mathrm{DE}$, apesar de ter sido menor que a da CPM, e não ter apresentado diferença estatística significativa. Numa meta-análise descrita por Frohelicher e cols. ${ }^{1}$ já se havia demonstrado que o DE elevou a acurácia do teste ergométrico para nível semelhante ao da CPM com Tálio, porém não maior do que a CPM com Tecnécio. Outro fator que poderia explicar essa similaridade estatística entre esses métodos é o de que a análise deste estudo se limitou a 12 meses, quando a maioria dos estudos do DE e CPM foram superiores a 3 anos. Uma placa aterosclerótica, mesmo vulnerável, nem sempre traz evento clínico num período de 12 meses, podendo instabilizar-se num período superior a este, dependendo do controle dos fatores de risco. Porém os métodos de estresse já poderiam detectar precocemente alterações 
como, por exemplo, no caso da CPM, de uma perfusão miocárdica deficitária. Ainda, propositadamente, foram escolhidos pacientes com dois ou mais fatores de risco para doença arterial coronariana, uma população mais suscetível a eventos e provavelmente com maior número de coronárias envolvidas. Sabidamente, o teste ergométrico apresenta maior sensibilidade em pacientes multiarteriais, o que poderia ter elevado a sensibilidade do DE. Porém o mesmo ocorre com a CPM e com qualquer outro método de estresse ${ }^{5}$. Por último, uma sensibilidade menor da CPM poderia ter sido causada pelo fato de que o paciente que consegue deambular e completar o teste ergométrico, possui melhor prognóstico do que os pacientes submetidos a teste farmacológico. O estudo GISSI-2 demonstrou em grupo de pacientes infartados, tratados com trombolíticos, que aqueles que não conseguiram deambular em esteira tiveram prognóstico pior do que os que completaram pelo menos o segundo estágio de Bruce ${ }^{6}$. Por isso não se analisou a sensibilidade da CPM na totalidade, mas somente dos pacientes que conseguiram fazer esteira, ou seja, os de melhor prognóstico.

A especificidade do $D E$, porém, foi muito baixa em relação à CPM, com significância estatística. Esse fato torna-se difícil de explicar, uma vez que Gianrossi e cols. ${ }^{7}$ demonstraram numa meta-análise uma especificidade maior que a sensibilidade do teste ergométrico. Um dado que chamou a atenção no nosso estudo foi o de que boa parte dos pacientes livres de eventos tiveram DE de risco intermediário, à custa de infradesnivelamento de segmento ST. Um estudo desenvolvido por Bugiardini e cols. $^{8}$ demonstrou em 42 mulheres, seguidas ao longo de 10 anos, todas com teste ergométrico alterado associado a defeitos de perfusão à CPM, porém com cateterismo normal, um alto risco de desenvolver doença arterial coronariana (lesão evidente em novo cateterismo), infarto agudo do miocárdio e morte. Assim, demonstrou-se que a alteração no teste ergométrico antecedeu em anos a lesão coronariana aterosclerótica. Apesar de este estudo ter tido um número de casos pequenos, lançou uma luz no entendimento do porquê encontrarmos tantos exames rotulados como "falsos-positivos" no teste ergométrico. Provavelmente a alteração de segmento ST, nesses casos, indicou que esses pacientes apresentem disfunção endotelial importante e com maior risco de eventos futuros.

Somente 3 pacientes apresentaram DE alto risco (todos os 3 desenvolveram evento em um ano). Por esse motivo, foram analisados com os do grupo intermediário. Atualmente, estão sendo desenvolvidos alguns estudos, em que se analisa se o valor do DE limite entre o grupo de pacientes intermediário para alto risco não estaria subvalorizado. Isto é, para que o paciente atinja o valor de alto risco (inferior a "-11"), este terá de apresentar alterações muito importantes de segmento ST e, geralmente, presença de angina de peito durante o teste ergométrico. Esses estudos indicarão, no futuro, qual seria o valor exato que deveremos considerar para o $\mathrm{DE}$ alto risco. Talvez um "valor de corte" bem maior que este (mais próximo de zero), já que indica alto risco. Em nosso estudo, por exemplo, demonstramos, como visto na figura 3 , que os pacientes de maior risco, que apresentaram evento ao longo de 12 meses, foram os que apresentaram DE inferior a zero $(<00)$, com alto significado estatístico. Outro ponto importante para corroborar esse fato foi o de que em nosso estudo mostramos um número relativamente grande de eventos nos pacientes com DE de risco intermediário.

A curva de Kaplan-Meier (fig. 5) deixa clara a diferença entre os escores de risco do DE com relação ao período livre de eventos, principalmente entre o DE alto risco para o baixo risco, demonstrando a confiabilidade desse

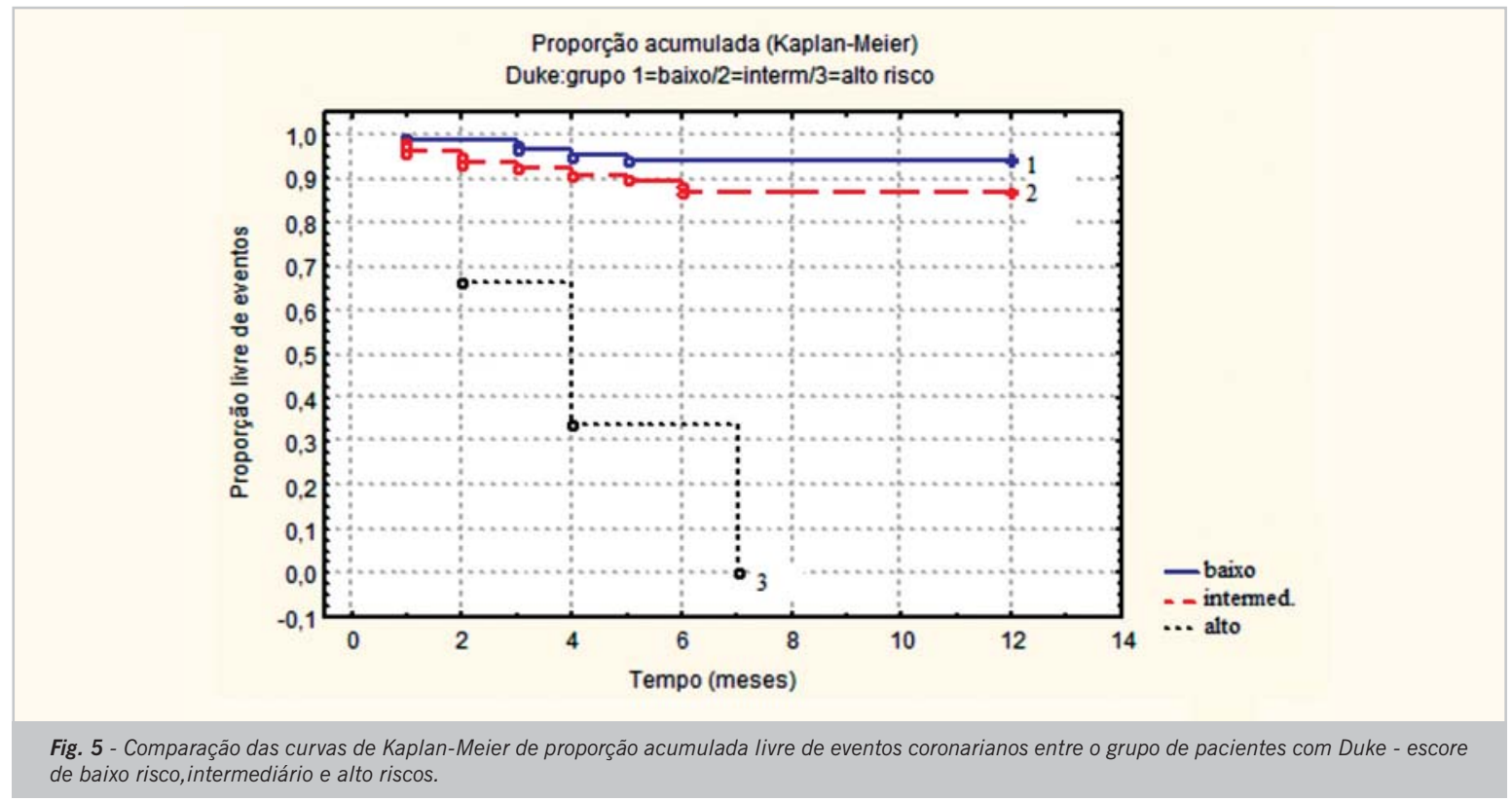


método em predizer risco de eventos. Apesar dessa confiabilidade, o DE possui limitações. Pacientes com infradesnivelamento de segmento ST já no traçado préteste, com idade superior a 75 anos, e em diabéticos, o DE demonstrou ter menor sensibilidade diagnóstica9 . Nesses casos, a associação com outros métodos de estresse pode beneficiar o paciente.

$\mathrm{Na}$ figura 4 registrou-se que os indivíduos com CPM normal tiveram menos de $5 \%$ de eventos no período deste estudo. Muitos destes apresentaram DE de risco intermediário. De fato, a CPM pode determinar quais dos pacientes, que apresentaram DE de risco intermediário, têm maior probabilidade de eventos coronarianos. Conforme citam Beller e cols. ${ }^{10}$, pacientes com DE intermediário que apresentaram CPM normal tiveram uma mortalidade inferior a $0,4 \%$ ao ano, enquanto entre os que apresentaram CPM alterada, a mortalidade foi de 8,9\% ao ano. A CPM teve grande importância na detecção dos indivíduos de maior risco, em conformidade com inúmeros estudos prévios ${ }^{11-13}$.

A principal limitação do nosso estudo foi o número relativamente pequeno de casos. Um estudo com maior número de casos poderia não só comprovar o nosso achado de forma mais eficaz, como também determinar os dados obtidos para vários subgrupos de pacientes. Por exemplo, poderíamos separá-los de acordo com os fatores de risco e determinar o DE de maior risco para cada um deles. Outra limitação, já mencionada, foi a análise do grupo de pacientes encaminhados à CPM, que conseguiram realizar a ergometria, ou seja, de melhor prognóstico. Isto pode ter interferido na análise comparativa entre os dois métodos de estresse. Por fim, outra limitação que vale lembrar deste estudo foi a análise subjetiva do examinador, tanto para determinação do segmento ST na ergometria, quanto da imagem da CPM. Para minimizar quaisquer erros, foram obtidos os dados por mais de um examinador e, principalmente, sem que a análise da ergometria tivesse conhecimento do resultado da CPM e vice-versa.

Concluindo o nosso estudo, o DE demonstrou ser tão sensível quanto a CPM na detecção de pacientes com maior risco de evento coronariano em 12 meses, principalmente os indivíduos que apresentaram DE inferior a zero, mesmo sendo assintomáticos. Muitos desses pacientes, classificados como de risco intermediário, devem receber atenção especial do clínico. Sugere-se que sejam encaminhados para investigação com outros métodos de estresse, como a CPM, para confirmação diagnóstica. Os que apresentaram DE de alto risco devem ser investigados imediatamente e, até mesmo, encaminhados para cineangiocoronariografia.

\section{Agradecimentos}

Aos técnicos de radiologia Avanildo Rubert, Patrícia Marina Piffar, Adair Obregão de Lima, João Maria da Silva, Roberto Ferreira da Silva, Leoni Matzenbacher, Sandro Afonso e às secretárias Sílvia Paula Colaço e Jocilene Raspzak Gorri à dedicação aos pacientes deste estudo e respeito com eles.

\section{Potencial Conflito de Interesses}

Declaro não haver conflitos de interesses pertinentes.

\section{REFERÊNCIAS}

1. Froelicher VF, Fearon WF, Fergusson CM, et al. Lessons learned from studies of the standard exercise ecg test. Chest. 1999; 116:1442-51.

2. Mark DB, Shaw L, Harrel FE, et al. Prognostic value of a treadmill exercise score in outpatients with suspected coronary artery disease. N Engl J Med. 1991;325:849-53.

3. Morrow K, Morris CK, Froehlicher VF, et al. Prediction of cardiovascular death in men undergoing noninvasive evaluation for coronary artery disease. Ann Intern Med. 1993;118:689-95.

4. Balady GK, Larson MG, Vasan RS, et al. Usefulness of exercise testing in the prediction of coronary disease risk among asymptomatic persons as a function of the Framingham Risk Score. Circulation. 2004;110:1920-5.

5. Tavel ME. Stress testing in cardiac evaluation. Current concepts with emphasis on the ECG. Chest. 2001;119:907-25.

6. Volpi A, de Vita C, Franzoni MG, et al. Predictors of nonfatal reinfarction in survivors of myocardial infarction after thrombolysis: results of the Gruppo italiano per lo studio della sopravvivenza nell' infarto miocardico (GISSI-2) data base. J Am Coll Cardiol. 1994;24:608-15.

7. Gianrossi R, Detrano R, Mulvihill D, et al. Exercise-induced ST depression in the diagnosis of coronary artery disease. A metaanalysis. Circulation. 1989; 80:87-98.
8. Bugiardini R, Manfrini O, Pizzi C, Fontana F, Morgagni G. Endothelial function predicts future development of coronary artery disease. A study of women with chest pain and normal coronary angiograms. Circulation. 2004;109:2518-23.

9. Kwok JM, Miller TD, Hodge DO, Gibbons RJ. Prognostic value of the Duke treadmill score in the elderly. J Am Coll Cardiol. 2002;39:1475-81.

10. Beller GA, Zaret BL. Contribution of nuclear cardiology to diagnosis and prognosis of patients with coronary artery disease. Circulation. 2000; 101:1465-78

11. Shaw LJ, Peterson ED, Shaw LK, et al. Use of a prognostic treadmill score in identifying diagnostic coronary disease subgroups. Circulation. 1998;98:1622-30

12. Lauer MS, Lytle B, Pashkow F, Snader CE, MarwickTH. Prediction of death and myocardial infarction by screening with exercise-thallium testing after coronary-artery-bypass grafting. Lancet. 1998;351:615-22.

13. Parisi AF, Hartingan PM, Folland ED. Evaluation of exercise thallium scintigraphy versus exercise electrocardiography in predicting survival outcomes and morbid cardiac events in patients with single- and double-vessel disease. Findings from the angioplasty compared to medicine (ACME) study. J Am Coll Cardiol. 1997;30:1256-63. 